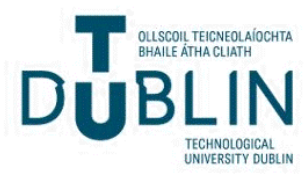

Technological University Dublin

ARROW@TU Dublin

2010-01-01

\section{A Diffusion Model for Spatially Dependent Photopolymerization}

\author{
Dana Mackey \\ Technological University Dublin, dana.mackey@tudublin.ie \\ Tzvetanka Babeva \\ Technological University of Dublin \\ Izabela Naydenova \\ Technological University of Dublin, izabela.naydenova@tudublin.ie
}

See next page for additional authors

Follow this and additional works at: https://arrow.tudublin.ie/cieoart

Part of the Condensed Matter Physics Commons, and the Optics Commons

\section{Recommended Citation}

Mackey, D. et al. (2010) A diffusion model for spatially dependent photopolymerisation. Progress in Industrial Mathematics at ECMI 2008. Mathematics in Industry, Vol. 15, Part 2, 253-258. doi:10.1007/ 978-3-642-12110-4_36

This Article is brought to you for free and open access by the Centre for Industrial and Engineering Optics at ARROW@TU Dublin. It has been accepted for inclusion in Articles by an authorized administrator of ARROW@TU Dublin. For more information, please contact arrow.admin@tudublin.ie, aisling.coyne@tudublin.ie, gerard.connolly@tudublin.ie.

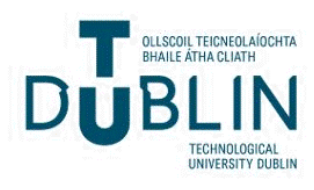




\section{Authors}

Dana Mackey, Tzvetanka Babeva, Izabela Naydenova, and VIncent Toal 


\title{
A diffusion model for spatially dependent photopolymerization
}

\author{
D. Mackey $^{1}$ \\ T. Babeva ${ }^{2}$ \\ V. Toal ${ }^{2}$ \\ I. Naydenova ${ }^{2}$ \\ ${ }^{1}$ School of Mathematical Sciences, Dublin Institute of Technology, Ireland \\ ${ }^{2}$ Centre for Industrial and Engineering Optics, Dublin Institute of Technology, Ireland
}

\begin{abstract}
Photopolymers represent an attractive class of optical recording materials due to properties such as high refractive index modulation, dry film processing, low cost, etc. Applications include holographic data storage disks, optical interconnections, memories and filters. This paper addresses the dynamics of short-exposure holographic grating formation; a new model is proposed to explain the experimental observations of low diffraction efficiency in high spatial frequency gratings.
\end{abstract}

\section{Introduction}

The basic formulation of a dry photopolymer system consists of one or two monomers, photoinitiator and sensitizing dye, all dispersed in a binder matrix. Upon uniform illumination, a monomer polymerizes and the refractive index of the system changes. When material is exposed to an interference pattern more monomers are being polymerized in the bright regions than in the dark ones. This sets up a concentration gradient and the monomer diffuses from dark to bright areas. The recorded holographic grating (spatial distribution of refractive index) is a result of changes in the relative density of components.

Grating evolution in photopolymers has been studied by several authors ([4], [6], etc.). However, the common feature of most theoretical models proposed to date is that they cannot describe the experimental observation of poor diffraction efficiency at high spatial frequencies. There are two theories explaining this poor response. The "nonlocal-response diffusion model" of [5] assumes growth of polymer chains away from their initiation locations and predicts that high frequency gratings can be improved if shorter polymer chains are created during the recording. Despite the successful theoretical model no supporting experimental evidence has been reported so far for spatial frequencies higher than 3000 lines $/ \mathrm{mm}$.

A second theory was proposed in [1] and [2]. It states that the counter diffusion of short-chain polymer molecules away from the bright fringes is responsible for the reduction 
in diffraction efficiency and predicts that producing short chains is not sufficient to achieve high spatial frequency resolution but, in addition, their diffusion must be suppressed. In order to achieve theoretical validation for this theory, we propose here a new mathematical model for hologram formation and compare numerical simulations of the refractive index modulation after short exposures with experimental results.

\section{Problem formulation}

The photopolymer is exposed to two coherent beams of intensities $I_{1}$ and $I_{2}$ which create the following illumination pattern

$$
I(x)=I_{0}(1+V \cos (k x)),
$$

where $k$ is the grating wavenumber, $I_{0}=I_{1}+I_{2}$ and $V=2 \sqrt{I_{1} I_{2}} /\left(I_{1}+I_{2}\right)$ are the overall intensity and visibility of the interference pattern, respectively. The holographic grating formation then proceeds in three steps: initiation, propagation and termination. Upon illumination, the sensitizing dye absorbs a photon and reacts with the electron donor to produce free radicals; in the presence of monomer these free radicals initiate polymerization. During the propagation step, free radicals and monomer molecules interact and produce growing polymer chains. At the termination step, two free radicals or two polymer chains interact and the polymer chains stop growing. As stated above, the faster consumption of monomer in the illuminated areas sets up a concentration gradient so the free monomer diffuses from dark to bright fringes; in addition we now assume that short-chain polymer molecules (or radicals) can also diffuse during recording. All these processes modify the spatial modulation of the refractive index and yield a phase grating.

The refractive index of a material consisting of a mixture of components can be calculated with the well-known Lorentz-Lorenz equation:

$$
\frac{n^{2}-1}{n^{2}+2}=\sum_{i} \Phi_{i} \frac{n_{i}^{2}-1}{n_{i}^{2}+2}
$$

where $n$ is the effective refractive index of the mixture, $n_{i}$ are the refractive indices of the components (monomer, polymer and binder) determined separately from spectrophotometric measurements, and $\Phi_{i}$ are the normalized concentrations of the components (e.g. $\Phi_{m}=m /(b+m+p)$, where $m, p$ and $b$ denote concentrations of monomer, polymer and binder, respectively). The details of this calculation are not important so will not be included here.

The refractive index modulation determines the grating strength and is calculated as the difference between the values in the bright and dark fringes,

$$
\Delta n(t)=n_{\max }(t)-n_{\min }(t)
$$

In short exposure conditions $\Delta n(t)$ should ideally exhibit fast growth followed by convergence to an equilibrium state. 


\section{Proposed model}

We now propose a generalization to existing models which takes into account monomer and polymer diffusion, creation of short polymer chains and introduces a simple "immobilization" mechanism which mimics the growth of polymer chains to the extent where they cannot diffuse any longer. The short exposure régime is also reflected in the model, whereby all polymerization and immobilization processes stop once the light beam is terminated.

In what follows, the spatial domain is assumed to be $x \in[0, \Lambda]$, where $\Lambda=\frac{2 \pi}{k}$ is the grating period (or fringe spacing). The classical model (see, for example, [6]) consists of a polymerization-diffusion equation for the monomer molecules but assumes diffusion stops once monomer is polymerized,

$$
\frac{\partial m}{\partial t}=D_{m} \frac{\partial^{2} m}{\partial x^{2}}-F(x) m .
$$

Here $m(x, t)$ denotes monomer concentration, $D_{m}$ is the monomer diffusion constant and the polymerization rate is proportional to the illumination

$$
F(x)=F_{0}(1+V \cos (k x))^{a} \equiv F_{0} f(x)
$$

where $F_{0}$ is the polymerization constant and $a>0$. The initial concentration of free monomer in the material is spatially uniform, $m(x, 0)=m_{0}$.

In addition, we now assume that short polymer chains can diffuse and the diffusion coefficient is also proportional to the illumination, $D(x)=D_{p} f(x)$, meaning that at high intensity, more short-chains are formed, which are more mobile. We also assume that short chains are converted to long chains at a rate proportional to monomer and polymer concentrations ( $\Gamma$ is the conversion rate constant) and that the long chains are immobile once formed. The resulting equations are

$$
\begin{aligned}
\frac{\partial m}{\partial t} & =D_{m} \frac{\partial^{2} m}{\partial x^{2}}-\Phi(t) F(x) m, \\
\frac{\partial p_{1}}{\partial t} & =\frac{\partial}{\partial x}\left[D(x) \frac{\partial p_{1}}{\partial x}\right]+\Phi(t)\left[F(x) m-\Gamma m p_{1}\right] \\
\frac{\partial p_{2}}{\partial t} & =\Phi(t) \Gamma m p_{1},
\end{aligned}
$$

where, $p_{1}(x, t)$ is the concentration of short polymer chains, $p_{2}(x, t)$ is the concentration of long polymer chains, with initial conditions $p_{1}(x, 0)=0, \quad p_{2}(x, 0)=0$. We assume these equations are supplemented by zero-flux boundary conditions. To account for a short exposure régime in equations (2)-(4) we have introduced the step function

$$
\Phi(t)= \begin{cases}1, & \text { if } t \leq t_{e} \\ 0, & \text { if } t>t_{e}\end{cases}
$$

where $t_{e}$ is the exposure time. Note that this model is only valid for exposure times which are much shorter than diffusion times, as otherwise diffusion coefficients for both monomers and polymers are known to be time dependent. 
With the choice of non-dimensional variables

$$
\bar{x}=\frac{x}{\Lambda}, \quad \bar{t}=t F_{0}, \quad \bar{m}=\frac{m}{m_{0}}, \quad \bar{p}_{i}=\frac{p_{i}}{m_{0}}(i=1,2),
$$

the model becomes

$$
\begin{aligned}
\frac{\partial m}{\partial t} & =\kappa \frac{\partial^{2} m}{\partial x^{2}}-\Phi(t) f(x) m \\
\frac{\partial p_{1}}{\partial t} & =\varepsilon \kappa \frac{\partial}{\partial x}\left[f(x) \frac{\partial p_{1}}{\partial x}\right]+\Phi(t)\left[f(x) m-\gamma m p_{1}\right] \\
\frac{\partial p_{2}}{\partial t} & =\Phi(t) \gamma m p_{1}
\end{aligned}
$$

where

$$
\kappa=\frac{D_{m}}{F_{0} \Lambda^{2}} ; \quad \varepsilon=\frac{D_{p}}{D_{m}} \ll 1 ; \quad \gamma=\frac{\Gamma m_{0}}{F_{0}} .
$$

We also have the initial and boundary conditions

$$
\begin{gathered}
m(x, 0)=1, p_{i}(x, 0)=0 \\
\frac{\partial m}{\partial x}(x, t)=\frac{\partial p_{1}}{\partial x}(x, t)=\frac{\partial p_{2}}{\partial x}(x, t)=0, \quad \text { for } x=0,1 .
\end{gathered}
$$

On adding and integrating equations (5)-(7) we get the conservation law

$$
\int_{0}^{1}\left[m(x, t)+p_{1}(x, t)+p_{2}(t)\right] d x=1,
$$

which is to be expected, since monomer is converted into polymer while the total concentration of particles remains constant.

\section{Results and conclusions}

The non-dimensional model (5)-(10) was integrated numerically using a standard finite difference method. The numerical values used for the diffusion constants are $D_{m}=10^{-7} \mathrm{~cm}^{2} / \mathrm{s}$, $D_{p}=10^{-9} \mathrm{~cm}^{2} / \mathrm{s}$ (close to the values determined in [3]), so $\varepsilon=0.01$. The polymerization rate constant is assumed to be $F_{0}=0.3 \mathrm{~s}^{-1}, a=0.5$, and $\Lambda$ is varied between $2 \cdot 10^{-7} \mathrm{~m}$ and $1 \cdot 10^{-5} \mathrm{~m}$ (corresponding to a range of $100-5000$ lines $/ \mathrm{mm}$ ). The exposure time is $t_{e}=0.2 \mathrm{~s}$, unless otherwise specified. Numerical values for $\gamma$ (the "immobilization" rate constant) would be difficult to determine experimentally; however we found that $\gamma=1$ yielded qualitatively and quantitatively satisfactory results.

Figures 1 and 2 show the evolution of the monomer and long polymer concentrations for various values of the system parameters. The spatial modulation of these species is represented over two grating periods. After the beam is stopped, the monomer and short polymer concentrations converge towards a homogeneous state under the influence 
of diffusion, while the spatial profile of the long polymer remains frozen. Note there is a departure from the expected sinusoidal pattern occurring for a combination of low spatial frequency and longer exposure time. A detailed analysis of how the ratio between diffusion and polymerization rates, $\kappa$, and the exposure time, $t_{e}$, affect the grating dynamics will form the subject of further study.
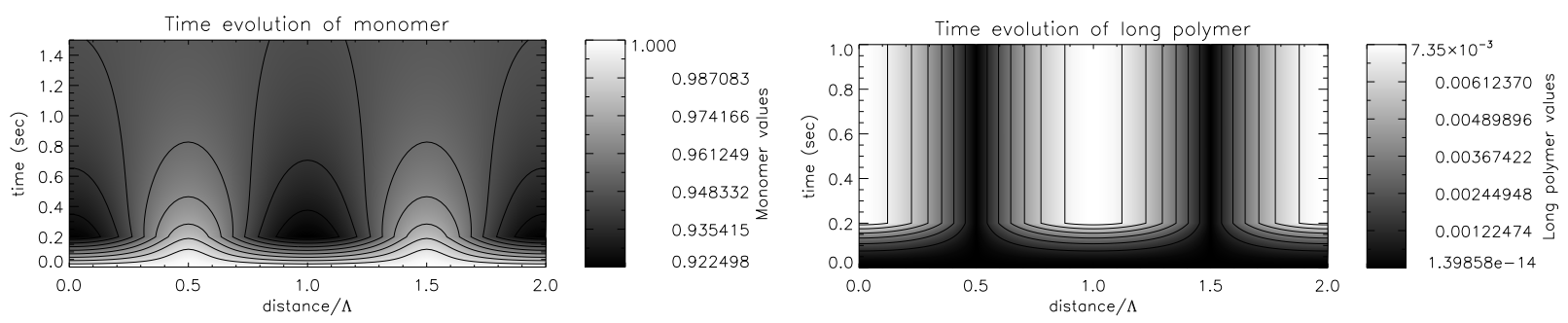

Figure 1: Relative concentrations of monomer $\left(\mathrm{m} / \mathrm{m}_{0}\right)$ and long polymer $\left(p_{2} / \mathrm{m}_{0}\right)$. Here $\Lambda=5 \cdot 10^{-6}, t_{e}=0.2 \mathrm{~s}$.
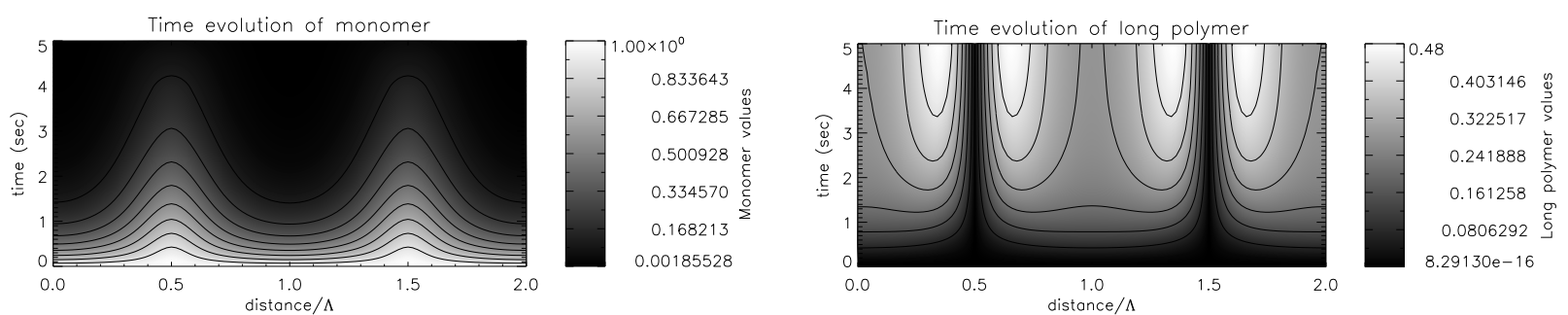

Figure 2: Relative concentrations of monomer $\left(\mathrm{m} / \mathrm{m}_{0}\right)$ and long polymer $\left(p_{2} / \mathrm{m}_{0}\right)$. Here $\Lambda=10^{-5} \mathrm{~m}$ and $t_{e}=4 \mathrm{~s}$.

Figure 3 shows remarkable agreement between the qualitative behaviour of the refractive index modulation obtained from experiment and model simulation, for three values of the spatial frequency $(200,350$ and 500 lines $/ \mathrm{mm})$. Figure 4 shows the time evolution of the refractive index modulation for a wider range of spatial frequencies, between 100 and 5000 lines $/ \mathrm{mm}$. Note that the model simulations reflect the experimental observations of a drop in refractive index modulation at high spatial frequencies.

In conclusion, the model validates the assumption that the poor high spatial frequency response in photopolymers can be explained by the diffusion of short polymer chains from bright to dark fringes. An improvement strategy would require that the holographic recording conditions be chosen so as to suppress the production of short polymer chains and low permeability binders be used in order to prevent their diffusion. Based on this principle, a successful experimental strategy for producing a high diffraction efficiency reflection hologram in an acrylamide-based photopolymer was recently presented in [3]. 

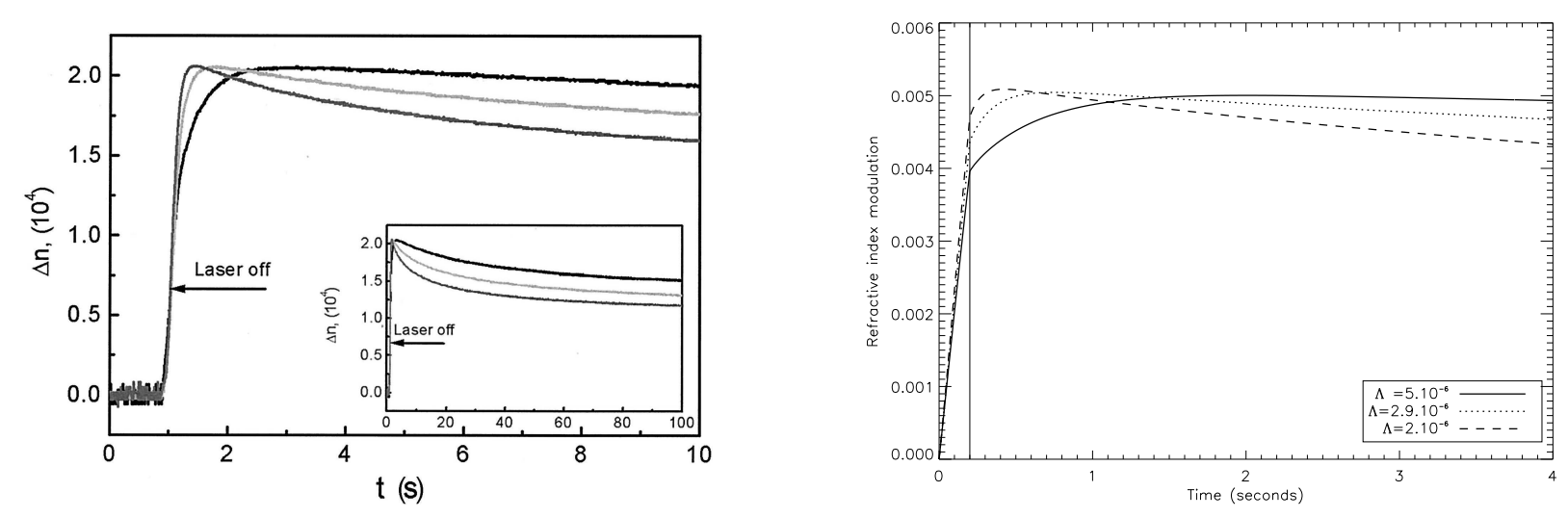

Figure 3: Comparison of experimental (left) and numerical (right) results for the refractive index modulation. The three curves correspond to $\Lambda=5 \cdot 10^{-6} \mathrm{~m}, \Lambda=2.9 \cdot 10^{-6} \mathrm{~m}$ and $\Lambda=2 \cdot 10^{-6} \mathrm{~m}$.

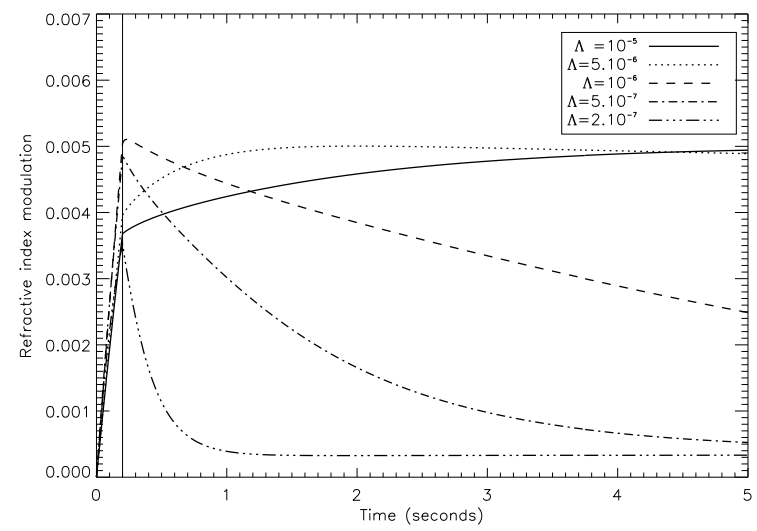

Figure 4: Refractive index modulation for several values of $\Lambda$. Note the deterioration of the grating strength for high spatial frequencies.

Acknowledgements: Financial support from the Science Foundation Ireland grant N 065/RFP/PHY085 is gratefully acknowledged.

\section{References}

[1] S. Martin, I. Naydenova, R. Jallapuram, R. Howard, and V. Toal, Proc. SPIE 6252, 62525-625217 (2006).

[2] I. Naydenova, R. Jallapuram, R. Howard, S. Martin and V. Toal, Appl. Opt. 43, 2900-2905 (2004) 
[3] I. Naydenova, R. Jallapuram, V.Toal and S. Martin, Appl. Phys. Lett., 92, 031109 (2008)

[4] S. Piazzola and B. Jenkins, J. Opt. Soc. Am. B 17, 1147-1157 (2000).

[5] T. Sheridan and R. Lawrence, J. Opt. Soc. Am. A, 17, 1108-1114, (2000).

[6] G. Zhao and P. Mourolis, J. Mod. Opt. 41, 1929-1939 (1994) 